\title{
Pharmacogenetics of cerebrovascular metabolism modulators in dementia due to Alzheimer's disease
}

\author{
Farmacogenética dos moduladores do metabolismo cerebrovascular na síndrome \\ demencial da doença de Alzheimer
}

Fabricio Ferreira de Oliveira

\begin{abstract}
Resumo da Tese de Doutorado apresentada à Escola Paulista de Medicina, Universidade Federal de São Paulo. Área de Neurologia e Neurociência. São Paulo SP, Brasil.

Correspondence: Fabricio Ferreira de Oliveira; Universidade Federal de São Paulo, Escola Paulista de Medicina, Departamento de Neurologia e Neurocirurgia; Rua Botucatu, 740, Vila Clementino; 04023-900 São Paulo SP, Brasil; E-mail: fabricioferreiradeoliveira@hotmail.com

Supervisor: Paulo Henrique Ferreira Bertolucci (Departamento de Neurologia e Neurocirurgia).

Co-Supervisora: Marilia de Arruda Cardoso Smith (Departamento de Morfologia e Genética).

Support: CAPES - Coordenação de Aperfeiçoamento de Pessoal de Nível Superior.

Received 20 October 2014; Received in final form 6 November 2014; Accepted 26 November 2014.
\end{abstract}

\begin{abstract}
The aims of this study were to investigate risk factors for cognitive and functional decline among 193 patients with Alzheimer's disease dementia (AD), and to conduct pharmacogenetic analysis on cerebrovascular metabolism modulators, taking into account APOE haplotypes and the genotypes of ACE, CETP, LDLR and the LXR- $\beta$ gene. For all patients, later age at AD onset was the most important risk factor for faster cognitive and functional decline, while the late-life coronary heart disease risk was inversely related to cognitive decline only for carriers of APOE4+ haplotypes. Schooling was protective against cognitive decline only for women and carriers of APOE4+ haplotypes, while higher body mass index in late life was protective against cognitive decline only for men. Carriers of the APOE- $\varepsilon 4 / \varepsilon 4$ haplotype had earlier AD onset, whereas genotypes of CETP and LDLR that had traditionally been associated with higher risk of AD were associated with later onset of dementia. Angiotensin-converting enzyme inhibitors caused a 50\% reduction in Mini-Mental State Examination score changes, and had better disease-modifying properties than did centrally-acting angiotensin-converting enzyme inhibitors alone. Angiotensin receptor blockers had genetically mediated effects that led to faster cognitive and functional decline, while patients with genetic tendencies towards faster cognitive and functional decline had maximum benefits when they used lipophilic statins, and vice versa.
\end{abstract}

Keywords: Alzheimer disease, dementia, cerebrovascular disorders, neurodegenerative diseases, risk factors, pharmacogenetics, genetics. 